\title{
A Comparative Study Between Silver Containing Dressings (Aquacel, Acticoat) and Sulfadiazine in Management of Superficial Partial Thickness Burns in Trunk and Extremities
}

Mohamed Mahfouz Mohamed, Amr Mohamed El Hefny, Mohamed Mamdouh Abdel Halim, Ahmed Saeed Saad, Wafaa Abdel Wahab Abdel Halim*

General surgery department of Ain-Shams University, Egypt

Plastic, reconstructive surgery and burns department of Ain-Shams University, Egypt

Plastic surgery and burns department of Om El-Masreen general hospital, Egypt

*Corresponding author: Wafaa Abdel Wahab Abdel Halim, General Surgery department of Ain Shams University, Egypt.

Received Date: August 13, 2020

Published Date: September 03, 2020

\begin{abstract}
The goal of this study is comparison between silver containing dressings (Aquacel or Acticoat) and Silver Sulfadiazine cream in management of superficial partial thickness burns according to time for complete wound healing, number of dressing changes, level of pain during dressing change, incidence of infection, and total cost effectiveness. The study found that silver containing dressings are superior to the conventional treatment with Sulfadiazine cream in superficial partial thickness burns. Key words: Superficial partial thickness burns, Sulfadiazine cream, Antimicrobial barriers, Acticoat, Aquacel Ag
\end{abstract}

\section{Introduction}

Burns are injuries of skin or other tissue caused by thermal, radiation, chemical, or electrical contact [1]. People of all ages are susceptible to minor burn injury. Second degree burns are common injuries in the outpatient department [2]. Burns exert a catastrophic influence on people in terms of human life, suffering, disability, and financial loss. The visible physical and the invisible psychological scars are long lasting and often lead to chronic disability [3]. Superficial partial thickness burns involve the epidermis and papillary dermis. Blisters occur within $24 \mathrm{~h}$ of injury. The exposed underlying reticular dermis is typically pink due to damage to dermal blood vessels, blanching, moist, and tender to touch. These burns usually heal within 2 to 3 weeks with minimal risk of scarring [4]. Superficial partial thickness burns characterized by pain due to exposed reticular layer of dermis [5]. Burn patients are at high risk for infection especially drug-resistant infection which often results in significantly longer hospital stays, delayed wound healing, higher costs, and higher mortality. Since the use of topical antibiotics, such as mafenide in the 1960s and silver sulfadiazine in the 1970s, and the early excision and grafting in the 1970s, thereafter, systemic infections and mortality have consistently decreased [6]. Silver and silver compounds have been routinely used as general antimicrobial agents, due to its effectiveness against a wide spectrum of bacterial or fungal pathogens, with lower risks to induce bacterial resistance 
compared to antibiotics [7]. Silver sulfadiazine still is frequently referred to as 'the gold standard' in the treatment of partial thickness burns because of its excellent antibacterial properties and its wide availability. However, in recent years, several reports have shown that this standard therapy also has a number of substantial disadvantages. Application of SSD always results in the formation of a pseudo- eschar layer on the burn wound which impairs evaluation of burn depth and healing status. Also, inactivation of much of the silver by wound fluid and formation of a pseudo- eschar[8] Silver sulfadiazine is relatively painless on application, has a high patient acceptance, and is easy to use, however daily dressing changes are labour intensive, expensive and induce fear and pain, especially in children. Post-burn pruritis is a common disadvantage of SSD, causes much distress, specifically in children. Uncontrolled scratching disrupts the re-epithelialization process and the fragile newly regenerated epithelium, in addition, the potential introduction of infective pathogens would also delay time to re-epithelialization (TTRE) [9].

Prolonged time until re-epithelialization is associated with increased burn depth and scar formation. To protect patients from the costly burden of managing burn scars, the current wound management approaches focus on shortening re-epithelialization time. Also, decreasing the number of dressing changes may reduce the potential pain associated with dressing changes. The advanced wound dressings containing silver are proposed as a new management option for local wound infections, different types of wound dressings have been designed for different purposes and functions. The ideal wound dressing maintains moisture in the wound, removes excess exudates, is non-adherent, prevents the entry of microorganisms, controls infection, allows gaseous exchange and leaves no foreign particles in the wound. The dressing itself is another source of bacterial contamination [10].

Acticoat is nanocrystalline silver dressings (made by Smith \&Nephew, UK) were developed to overcome some of the shortcomings of the traditional silver containing dressings. Silver release is triggered by hydration of the dressing with sterile water. The outer silver layer is designed to provide antimicrobial protection whilst the inner core helps to maintain a moist environment. Nanocrystalline silver (NCS) is a broad spectrum antimicrobial and antifungal agent. Acticoat has also been shown to inhibit biofilm formation for Pseudomonas aeruginosa and Acinetobacter baumannii by more than $90 \%$ in vitro. There are a few reports in the literature of Acticoat being used in combination with such dressings. Hydrogels (the Intrasite gel) are sometimes used since the regular moistening of Acticoat can be labour intensive and impractical. If some of these dressings can be used in combination, we can combine their different attributes to achieve a synergistic effect. The antimicrobial effect of Acticoat is up to 7 days [11].

Aquacel is the first hydrofiber dressing to be released by (ConvaTec, UK) in 1997. Aquacel Ag dressing is a hydrofiber dressing that consists of sodium carboxmethyl cellulose, which forms a gel on contact of body fluid, which contains broad spectrum antimicrobial properties. Aquacel Ag+ Extra is an example to nextgeneration antibiofilm silver dressing (NGAD). Few dressings have been designed specifically to facilitate reduction and subsequent prevention of wound biofilm. NGAD containing ionic silver and antibiofilm agents has been introduced, with evidence of favorable clinical outcomes. The antimicrobial effect of Aquacel Ag extra is up to 14days [12].

\section{Patients and Methods}

this pilot study was carried out as prospective, Comparative, Interventional, Clinical trial for six months started from March 2020. It involved 20 patients with superficial partial thickness burns in trunk and extremities. The patients were divided into 2 groups; Group (A):10 patients were dressed by Silver containing dressings (Aquacel Ag or Acticoat). Group(B):10 patients were dressed by sulfadiazine.

\section{Inclusion criteria}

i. Both males and females.

ii. Age group 10-60 years old

iii. Second degree superficial partial thickness burns including trunk and extremities.

\section{Exclusion criteria}

i. Patients with history of diabetes, peripheral vascular disease, clotting disorders, or any illness that would impair assessment of healing.

ii. Patients with collagen disorders, other acute or chronic dermatological disorders.

iii. Pregnant patients.

iv. Burns covering large area (more than 20\% TBSA burn)

v. Allergy to Sulfadiazine or silver products.

All patients were subjected to; primary assessment of patients; Airway assessment included visualizing the upper airway to look for evidences of burn, Breath sounds were auscultated, Vital signs, the Glasgow Coma Scale (GCS) was used to trend the patient's neurologic status throughout resuscitation and to determine any associated injury, and skin exposure and removing of all of the patient's clothes, jewelry, and shoes to stop the burning process.

Secondary assessment of patients; History was gathered from the patient, family members, or those who were at the scene, lab work and tests within the first 24 hours of a patient's admission, pre-medicating the patient with an analgesic agents ,TBSA $\%$ and burn depth were assessed. Steps of intervention technique; For both groups at the 1st visit; photos of the burn wounds to act as baseline for follow up of healing, and wound swabs from the surface of burns, were taken. Also, assessment of pain during and after dressing application, using Wong-Baker Faces- Pain-Scale.

In group (A); the wounds were cleaned with normal saline; the area was covered with silver dressings (Aquacel Ag or Acticoat) then bandaged. 
In group (B); the wounds were cleaned with normal saline, and then the area was covered with Sulfadiazine cream and dry sterile gauze then bandaged. The dressing was changed every day. The standard SSD creams used in this study were Hyalo4 Control and Dermazin creams. Post intervention surveillance: follow up of the patients to evaluate healing rates, incidence of wound infection, and pain scales.

\section{Cases Presentation}

\section{Case 1}

42 years old male patient with 3\%superficial partial thickness flame burn of right hand (Figure 1).

\section{Case 2}

15 years old female with $2 \%$ superficial partial thickness scald burn at right lower leg (Figure 2).

\section{Case 3}

33 years old male with 7\%superficial partial thickness flame burn of right leg (Figure 3).

\section{Case 4}

10 years old female with $3 \%$ superficial partial thickness scald burn of right side of chest (Figure 4).

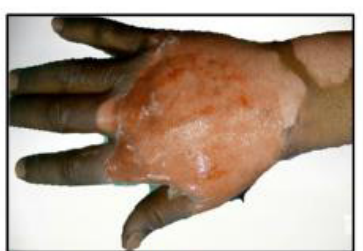

Day 1 post burn

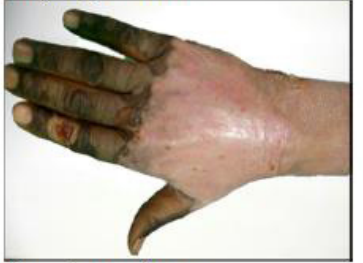

Day 9 post burn

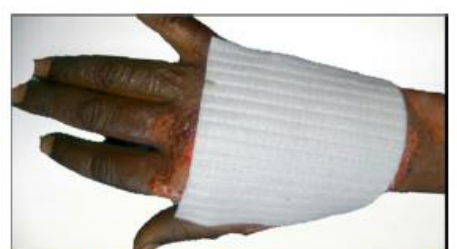

Aquacel Ag dressing

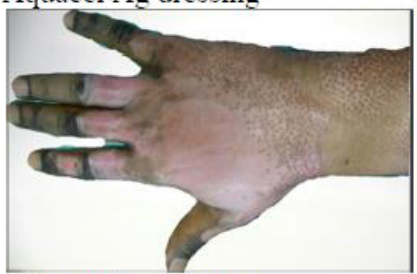

Day 14 post burn

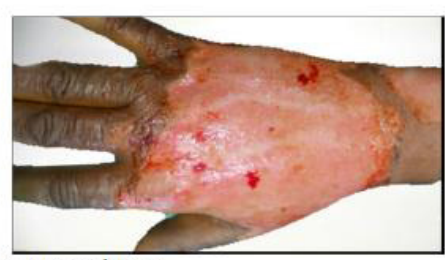

Day 4 post burn

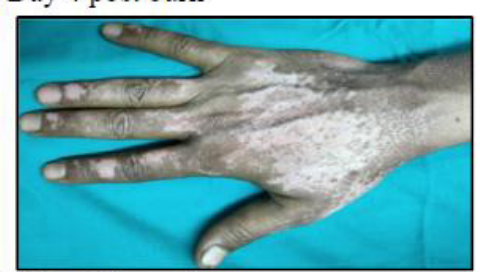

Day 25 post burn

Figure 1.

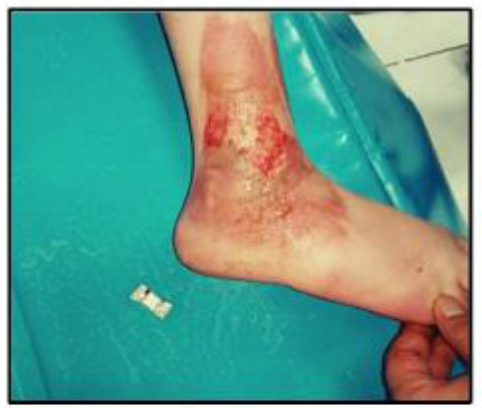

Day 1 post burn

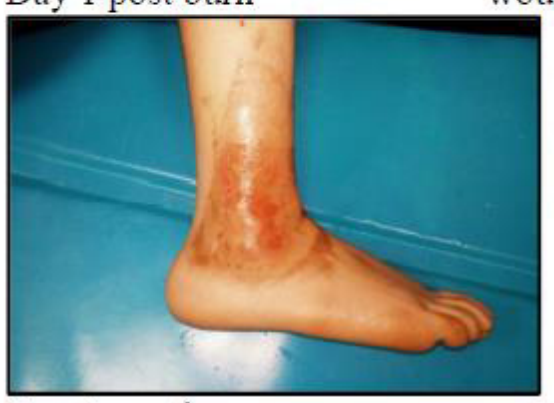

Day 6 post burn

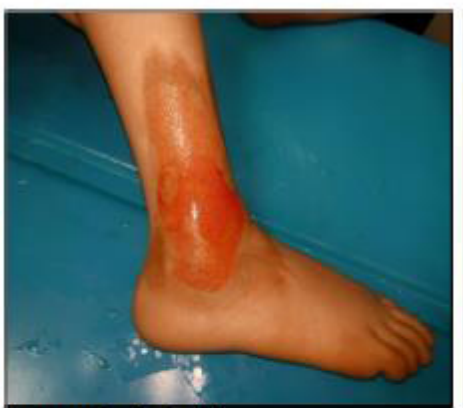

wound debridement

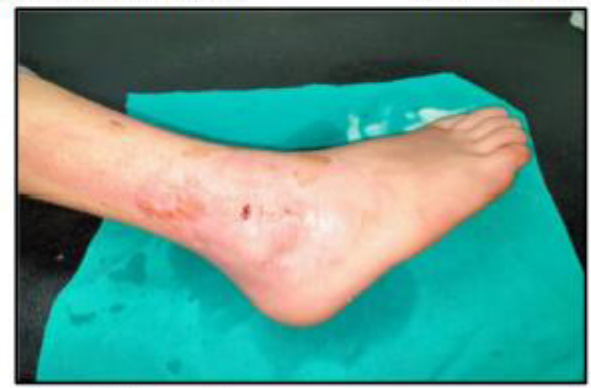

Day 14 post burn

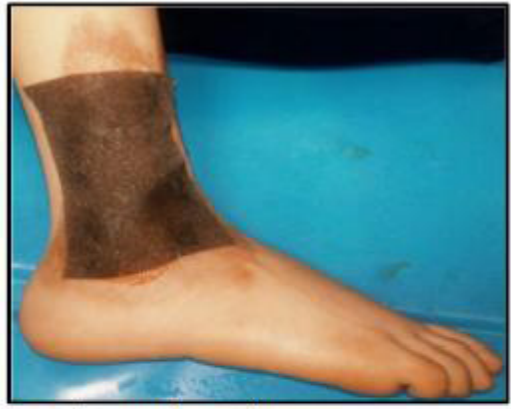

Acticoat dressing

Figure 2. 

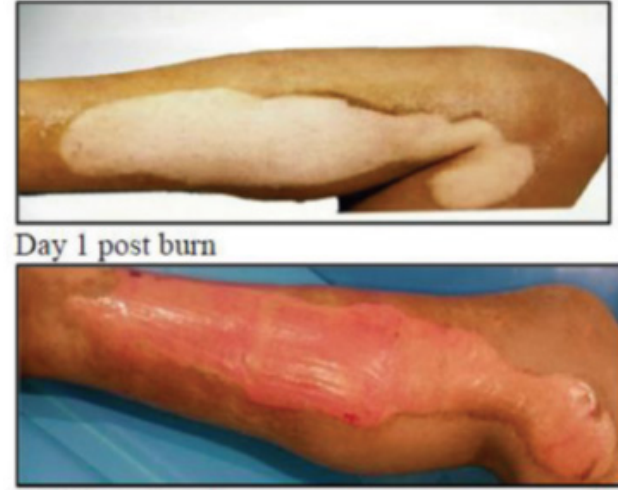

Day 6 post burn

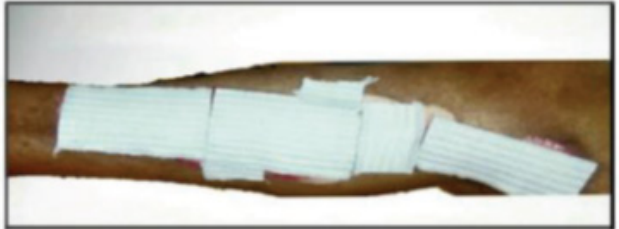

Aquacel $\mathrm{Ag}$ dressing

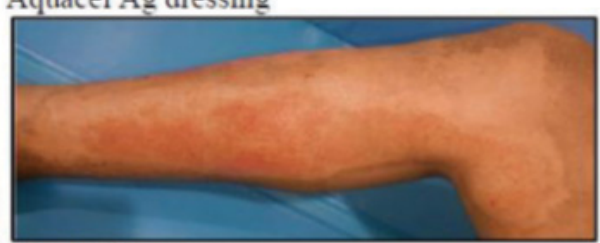

Day 12 post burn

Figure 3.

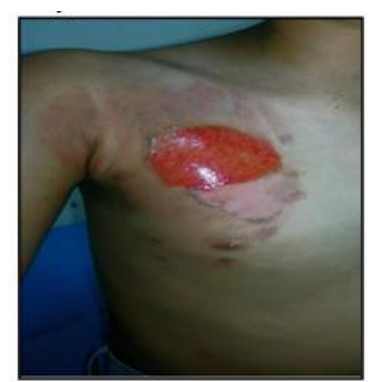

Day 1 post burn

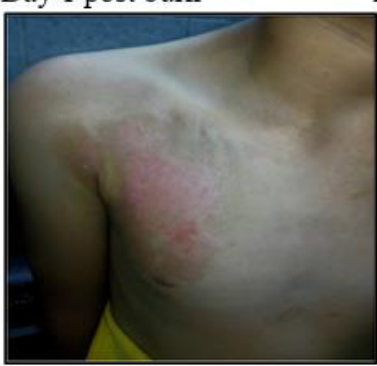

Day 10 post burn

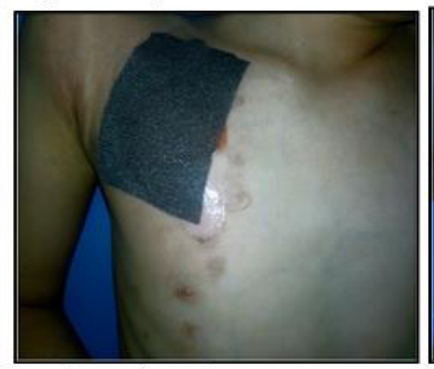

Acticoat dressing

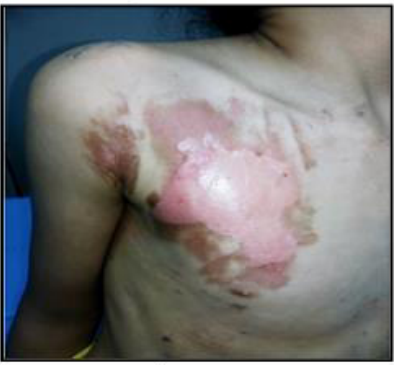

Day 6 post burn

Figure 4.

\section{Statistical Methodology}

This is a prospective, randomized trial involving patients with partial thickness burns less than 20\% (TBSA\%) in trunk and extremities.

The study cases were allocated into two groups according to the used topical antimicrobial agents.

Table 1: Demographic data of all patients.
Group (A): 10 patients were dressed by Silver containing dressings (Aquacel Ag or Acticoat).

Group (B): 10 patients were dressed by sulfadiazine cream.

\section{Results}

(Table 1,2,3) (Figure 5) (Table 4,5) (Figure 6,7) (Table 6,7)

\begin{tabular}{|c|c|c|}
\hline \multicolumn{2}{|c|}{} & Total no. = 20 \\
\hline \multirow{2}{*}{ Age } & Mean \pm SD & $28.15 \pm 13.28$ \\
\cline { 2 - 3 } & Range & $10-55$ \\
\hline \multirow{2}{*}{ Sex } & Female & $9(45.0 \%)$ \\
\cline { 2 - 3 } & Male & $11(55.0 \%)$ \\
\hline \multirow{2}{*}{ TBSAB\% } & Median (IQR) & $5.50(4.5-8)$ \\
\cline { 2 - 3 } & Range & $2-15$ \\
\hline \multirow{2}{*}{ Type of burn } & Scald & $11(55.0 \%)$ \\
\cline { 2 - 3 } & Flame & $9(45.0 \%)$ \\
\hline
\end{tabular}


Table 2: Demographic data for both groups

\begin{tabular}{|c|c|c|c|c|c|}
\hline & & Silver containing dressing group & Sulfadiazine group & \multirow{2}{*}{ Test value } & \multirow{2}{*}{ P-value } \\
\hline & & No. $=10$ & No. $=10$ & & \\
\hline \multirow{2}{*}{ Age } & Mean \pm SD & $27.20 \pm 13.20$ & $29.10 \pm 14.00$ & \multirow{2}{*}{$-0.312 \bullet$} & \multirow{2}{*}{0.758} \\
\hline & Range & $10-47$ & $10-55$ & & \\
\hline \multirow{2}{*}{ Sex } & Female & $4(40.0 \%)$ & $5(50.0 \%)$ & \multirow{2}{*}{$0.202^{*}$} & \multirow{2}{*}{0.653} \\
\hline & Male & $6(60.0 \%)$ & $5(50.0 \%)$ & & \\
\hline \multirow{2}{*}{ TBSAB $\%$} & Median (IQR) & $5.5(4-8)$ & $5.5(5-8)$ & \multirow{2}{*}{$-0.267 \neq$} & \multirow{2}{*}{0.789} \\
\hline & Range & $2-10$ & $2-15$ & & \\
\hline
\end{tabular}

P-value > 0.05: Nonsignificant; P-value < 0.05: Significant; P-value < 0.01: Highly significant *: Chi-square test; $\bullet:$ Independent t-test; $\neq:$ : MannWhitney test

Table 3: Type of dressing used in all patients.

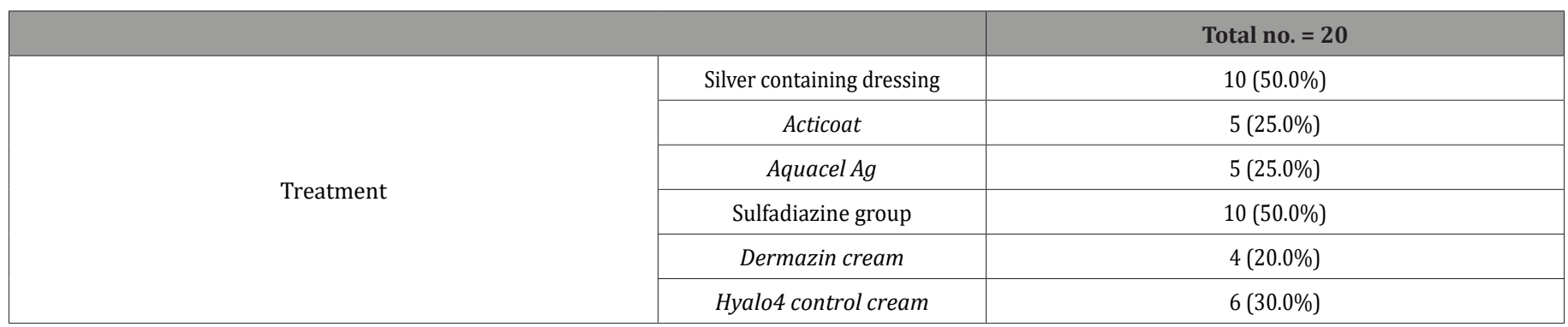

Table 4: Healing rates for both groups.

\begin{tabular}{|c|c|c|c|c|c|}
\hline & & Silver containing dressing group & Sulfadiazine group & & \\
\hline & & No. $=10$ & No. $=10$ & Test value & P-value \\
\hline \multirow[b]{2}{*}{ Healing rate (days) } & Mean \pm SD & $12.44 \pm 2.24$ & $15.60 \pm 4.95$ & \multirow[b]{2}{*}{$-1.754 \bullet$} & \multirow[b]{2}{*}{0.097} \\
\hline & Range & $10-16$ & $11-25$ & & \\
\hline
\end{tabular}

P-value > 0.05: Nonsignificant; P-value < 0.05: Significant; P-value < 0.01: Highly significant *: Chi-square test; $\bullet:$ Independent t-test; $\neq$ : Mann-Whitney test

Table 5: Pain scale results for both groups.

\begin{tabular}{|c|c|c|c|c|c|}
\hline & & Silver containing dressing group & Sulfadiazine group & \multirow{2}{*}{ Test value } & \multirow{2}{*}{ P-value } \\
\hline & & No. $=10$ & No. $=10$ & & \\
\hline \multirow{2}{*}{ Pain $1^{\text {st }}$ visit } & Median (IQR) & $5(4-5)$ & $7(6-7)$ & \multirow{2}{*}{$-3.481 \neq$} & \multirow{2}{*}{0.000} \\
\hline & Range & $3-6$ & $5-8$ & & \\
\hline \multirow{2}{*}{ Pain $2^{\text {nd }}$ visit } & Median (IQR) & $3(2-3)$ & $5(4-6)$ & \multirow{2}{*}{$-3.276 \neq$} & \multirow{2}{*}{0.001} \\
\hline & Range & $1-4$ & $3-7$ & & \\
\hline \multirow{2}{*}{ Pain $3^{\text {rd }}$ visit } & Median (IQR) & $0(0-0)$ & $2.5(2-4)$ & \multirow{2}{*}{$-3.626 \neq$} & \multirow{2}{*}{0.000} \\
\hline & Range & $0-1$ & $1-6$ & & \\
\hline
\end{tabular}

P-value > 0.05: Nonsignificant; P-value < 0.05: Significant; P-value < 0.01: Highly significant *: Chi-square test; $\neq$ : Mann- Whitney test.

Table 6: Incidence of infection in 1st and 2nd swabs for both groups.

\begin{tabular}{|c|c|c|c|c|c|}
\hline & & Silver containing dressing group & Sulfadiazine group & \multirow{2}{*}{ Test value } & \multirow{2}{*}{ P-value } \\
\hline & & No. $=10$ & No. $=10$ & & \\
\hline \multirow{2}{*}{ Swab 1st visit } & No & $10(100.0 \%)$ & $9(90.0 \%)$ & \multirow{2}{*}{$1.053^{*}$} & \multirow{2}{*}{0.305} \\
\hline & E.coli & $0(0.0 \%)$ & $1(10.0 \%)$ & & \\
\hline \multirow{3}{*}{ Swab 2nd visit } & No & $9(90.0 \%)$ & $8(80.0 \%)$ & \multirow{3}{*}{$3.059^{*}$} & \multirow{3}{*}{0.217} \\
\hline & Pseudomonas & $0(0.0 \%)$ & $2(20.0 \%)$ & & \\
\hline & Lost & $1(10.0 \%)$ & $0(0.0 \%)$ & & \\
\hline
\end{tabular}

P-value $>0.05$ : Nonsignificant; P-value $<0.05$ : Significant; P-value $<0.01$ : Highly significant *: Chi-square test. 
Table 7: No. of dressings and visits for both groups.

\begin{tabular}{|c|c|c|c|c|c|}
\hline & & Silver containing dressing group & Sulfadiazine group & \multirow{2}{*}{ Test value } & \multirow{2}{*}{ P-value } \\
\hline & & No. $=10$ & No. $=10$ & & \\
\hline \multirow{2}{*}{ No. of dressings } & Median (IQR) & $2(1-2)$ & $13(10-20)$ & \multirow{2}{*}{$-3.830 \neq$} & \multirow{2}{*}{0.000} \\
\hline & Range & $1-3$ & $6-24$ & & \\
\hline \multirow{2}{*}{ No. of visits } & Median (IQR) & $3(2-3)$ & $4(4-5)$ & \multirow{2}{*}{$-3.178 \neq$} & \multirow{2}{*}{0.001} \\
\hline & Range & $1-4$ & $3-6$ & & \\
\hline
\end{tabular}

P-value > 0.05: Nonsignificant; P-value < 0.05: Significant; P-value $<0.01$ : Highly significant $\neq$ : Mann-Whitney test

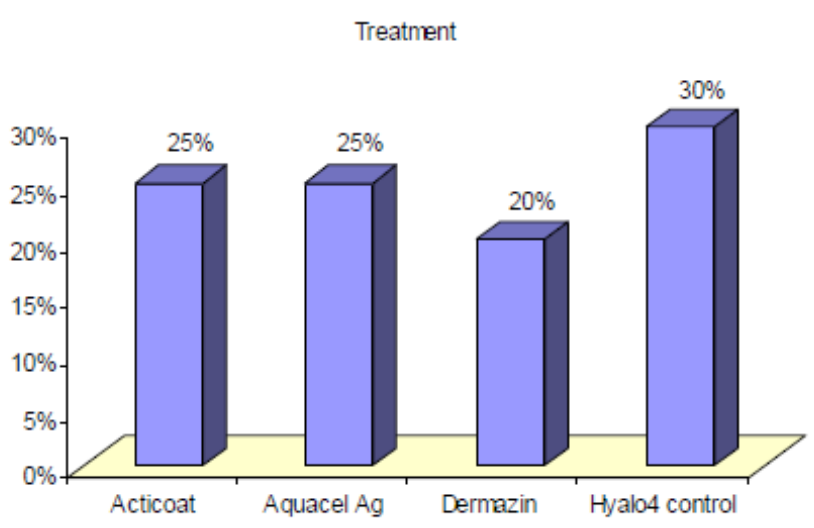

Figure 5: Type of dressings for both groups.

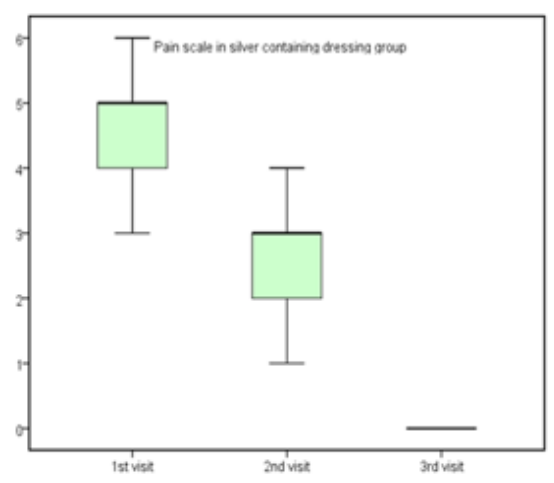

Figure 6.

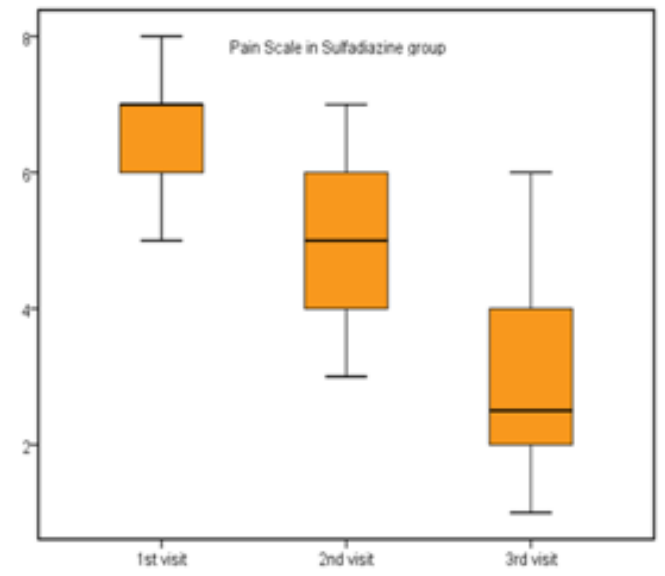

Figure 7. 


\section{Discussion}

This study was carried out on $n=20$ of patients. 17 (85\%) were outpatients and $3(15 \%)$ were inpatients. Of the total number of patients, 9 females (45.0\%) and 11 males (55.0\%), with 11 scald $(55.0 \%)$ and 9 flame (45.0\%) superficial partial thickness burns. The range of number of visits for group (A) was (1 to 4) day and the range for group (B) was (3 to 6) day. The mean of healing rates for silver dressings group was $(12.44 \pm 2.24)$ with range from (10 to 16$)$ days and for sulfadiazine group was $(15.60 \pm 4.95)$ with range from (11 to 25) days. The incidence of infection in Sulfadiazine group was $20 \%$ (2 patient infected with Pseudomonas) in comparison to silver dressings group with no infection burden. Using Wong-Baker faces pain scale to assess pain for both groups at the 1st, 2nd, and 3 rd visits, the median was for group (A); $(5,3,0)$ and for group (B); $(7,5,2.5)$ respectively.

Number of dressing was highly significant to silver containing dressings group (P-value $=0.000)$, for group $(\mathrm{A})$, the range of no. of dressings was (1 to 3 ) and for group (B), the range of no. of dressings was (6 to 24). According to total cost in EGP, the median (IQR) for group(A) was 300(250-500) EGP, and for group (B) was 495(200-1080)EGP. 40\% (4 patients) in sulfadiazine group needed intrasite gel for debridement of pseudo-eschar, and 10\% (1 patient) in the same group needed Mebo cream as additional treatments with (median=164.00 $\pm 83.55 \mathrm{EGP}$ ) to the 5 patients. According to study complications, there was one patient lost after first dressing in silver dressings group, between the rest 9 patients in this group 1 patient (10\%) had itching sensation on the 12th day post burn, and (1) patient had post burn scar. In Sulfadiazine cream group with total number 20 patients, 4 patients (40\%) had pseudoeschar on the burn surfaces during dressings, 2 patients (20\%) had itching sensation, and one of them (10\%) itching complicated with ulceration.

\section{Study limitations}

Poor patient compliance, irregular visits; patient's dropouts, were the common obstacles in this study.

\section{Conclusion}

We have found a faster wound healing process, less number of dressing changes, lower pain scores both during and after the wound dressing procedures, more patients' satisfaction and lower susceptibility to complications in the silver containing dressings group (Acticoat or Aquacel $\mathrm{Ag}$ ) than in the conventional group which received the usual wound care with silver Sulfadiazine cream reflecting the positive impact of silver dressings on the wound healing process.

\section{Acknowledgement}

None.

\section{Conflict of Interest}

No conflict of interest.

\section{References}

1. McLafferty E, Hendry C, Farley A (2012) The integumentary system: anatomy, physiology, and function of skin. Nurs Stand 27(3): 35-42.

2. O'Brien SP, Billmire DA (2008) Prevention and management of outpatient pediatric burns. J Craniofac Surg 19(4): 1034-1039.

3. Lawrence JW, Fauerbach JA, Thombs BD (2006) Frequency and correlates of depression symptoms among long-term adult burn survivors. Rehabilitation Psychology 51(4): 306-313.

4. Shehan H, Peter D (2005) ABC of burns: Pathophysiology and types of burns. B M J 328(7453): 1427-1429.

5. Mendes BB, Gómez Florit M, Babo PS, Domingues RM, Reis RL, et al. (2018) Blood derivatives awaken in regenerative medicine strategies to modulate wound healing. Advanced Drug Delivery Reviews 129: 376393.

6. Rowan Matthew P, Leopoldo C, Eric A Elster, David M Burmeister, Lloyd F Rose, et al. (2015) Burn Wound Healing and Treatment: Review and Advancements. Critical Care 19:243.

7. Klasen $H$ (2000) A historical review of the use of silver in the treatment of burns II. Renewed interest for silver. Burns 26(2): 131-138.

8. Fuller FW (2009) The Side Effects of Silver Sulfadiazine. J Burn Care Res 30(3): 464-470.

9. Vloemans AFPM, Hermans MHE, van der Wal MBA, Liebregts J, Middelkoop E (2014) Optimal treatment of partial thickness burns in children: A systematic review. Burns 40(2): 177- 190.

10. Khundkar R, Malic C, Burge T (2010) Use of Acticoat ${ }^{\mathrm{TM}}$ dressings in burns: What is the evidence? Burns 36(6): 751-758.

11. Verbelen J, Hoeksema H, Heyneman A, Pirayesh A, Monstrey S (2014) Aquacel ${ }^{\circledR}$ Agdressing versus Acticoat ${ }^{\mathrm{TM}}$ dressing in partial thickness burns: A prospective, randomized, controlled study in 100 patients. Part 1: Burn wound healing. Burns 40(3): 416-427.

12. Blanchette V, Belosinschi D, Lai TT, Lyne Cloutier, Simon Barnabé (2020) New Antibacterial Paper Made of Silver Phosphate Cellulose Fibers: A Preliminary Study on the Elimination of Staphylococcus aureus Involved in Diabetic Foot Ulceration. Biomed Res Int 2020:1304016. 